HEARES 01759

\title{
Cochlear electrode reimplantation in the guinea pig
}

\author{
Adena B. Greenberg, Michael W. Myers, Duane O. Hartshorn, Josef M. Miller \\ and Richard A. Altschuler
}

The Unitersity of Michigan, Kresge Hearing Research Institute, Ann Arbor, Michigan, USA

(Received 23 May 1991; Revision received 14 January 1992; Accepted 9 March 1992)

\begin{abstract}
Cochlear implants are being applied to an ever widening patient population, including children in whom lifetime use of these devices is anticipated. Replacement of implants can be expected for reasons of device failure as well as future upgrading. This investigation was undertaken to examine the effect of cochlear electrode explantation and reimplantation on spiral ganglion cell survival. Guinea pigs with normal ears were initially implanted and either explanted or explanted and reimplanted (at 2 months) with a single wire ball-tip intracochlear electrode or a silastic carrier (each remaining for an additional 2 months). Little loss of hair cells or auditory nerve was observed across experimental groups and normal controls. Restricted basal turn cochlear and spiral ganglion cell loss was observed in a few animals in each group and was likely associated with mechanical damage from initial implantation. Likewise the scattered organ of Corti damage and hair cell loss observed was noted in only a few cochleae in each experimental group. Therefore, no significant differences in the average pathology across experimental groups and controls were observed. Thus, explantation or explantation with subsequent reimplantation does not appear to constitute an additional significant pathological risk compared to implantation alone.
\end{abstract}

Cochlear prosthesis; Spiral ganglion cell; Electrode reimplantation; Safety; Pathology

\section{Introduction}

With the increasing application of cochlear implants to a larger population of patients with profound hearing loss, the need for improving and understanding the safety of cochlear implants is paramount. All current devices have a limited life span and have demonstrated periodic device failure, necessitating occasional replacement. This issue is clearly relevant to the implantation of children, in whom the intracochlear device might be needed over a period of seventy or more years. Additionally, as the design of implanted devices is improved, it may become appropriate to upgrade an individual's system. However, there may be special risks associated with removal and reimplantation. It has been shown that cochlear implant performance is correlated with the number of remaining spiral ganglion cells (Pfingst et al., 1985). If significant damage occurs due to explanting and reimplanting an electrode system, a patient may prefer to wait for the introduction of an improved prosthesis prior to initial implantation. On the other hand, such a decision could allow further nerve degeneration in ears with receptor damage, since nerve loss appears to increase with time

Correspondence to: Josef M. Miller, Kresge Hearing Research Institute, The University of Michigan, 1301 E. Ann Street, Ann Arbor, MI 48109-0506, USA.
(Webster et al., 1981; Jyung et al., 1989). Indeed, given recent data indicating that early stimulation of the deafened auditory system may preserve spiral ganglion cells and auditory nerve fibers (Lousteau, 1987; Leake et al., 1989; Hartshorn et al., 1991; Miller et al., 1991), it may be optimal to implant early after deafness is identified.

Numerous investigations have defined the risks associated with implantation of an intracochlear device (Otte et al., 1978; Leake et al., 1985; Leake-Jones et al., 1983; Schindler et al., 1974, 1976, 1979). They are primarily associated with mechanical trauma due to surgical manipulation. These risks, although generally considered acceptable, consititute a clear morbidity associated with all cochlear implants. It is reasonable to presume that explantation and reimplantation will multiply these risks and may introduce new ones. However, this question has not been adequately addressed.

In a preliminary study designed to evaluate the histological effect of reimplantation, Miller et al. (1987) implanted the normal ears of four monkeys (7 ears) using either a single wire ball-tipped electrode or a multicontact electrode array. At 10 months ball-tip electrodes were replaced with either new ball-tip electrodes or multicontact implants. Histological evaluation at 16 months revealed selective loss of spiral ganglion cells in the cochlear apex of re-implanted animals. However, the small sample size $(N=7)$ makes the significance difficult to clarify. 

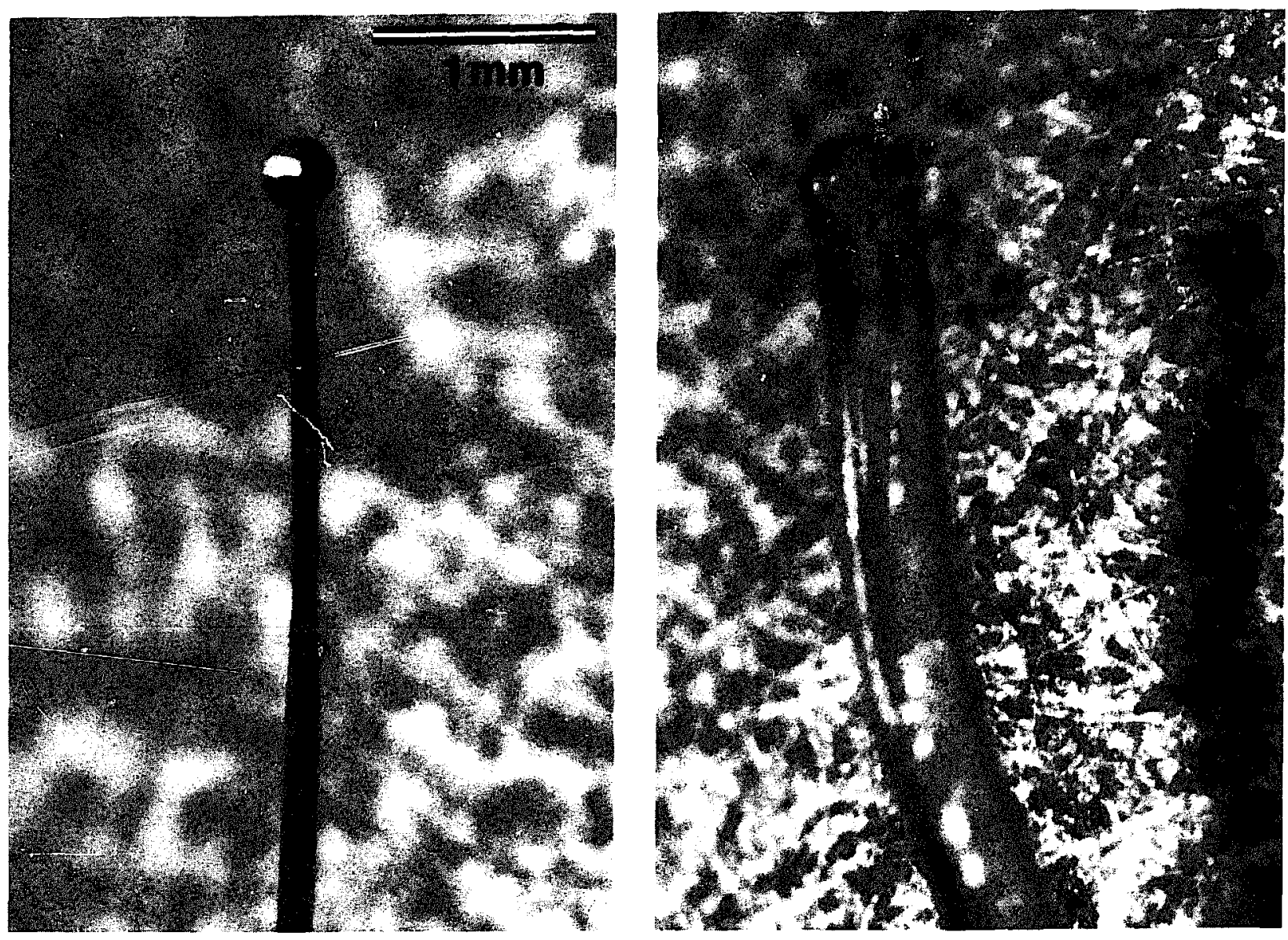

Fig. 1. Photograph of ball-tip cochlear implant (left) and silastic carrier implant (right).

The current study was undertaken in an attempt to determine whether the spiral ganglion cell loss observed in the primate can be verified in the rodent and if so, to determine if the damage is based upon reimplantation or if it is present with only explantation. Moreover, for reasons of upgrading we examined whether risks associated with reimplantation are similar with both single and multielectrode devices.

\section{Materials and Methods}

Thirty-three pigmented, Hartley strain guinea pigs (250-300 g) with intact Preyer's reflexes were used in this investigation. All surgical procedures were performed using aseptic techniques and conformed to guidelines of humane animal care. Two types of cochlear electrodes were used (Fig. 1). A single Teflon coated 5T Pt-Ir wire with a $250 \mu \mathrm{m}$ diameter ball-tip was used to simulate a single channel electrode. A simple silastic carrier with a central stabilizing wire but no external electrodes was used to simulate a multielectrode system. The implants terminated outside the middle ear against the mastoid bulla.

The animals were divided into five experimental groups (Fig. 2). Group one animals were implanted with a ball-tip electrode and examined at four months. Group two animals were implanted with a ball-tip electrode, explanted at two months, and examined at four months. Group three animals were implanted with a ball-tip electrode, explanted and reimplanted with a new ball-tip electrode at two months, and examined at four months. Group four animals were implanted with a ball-tip electrode, explanted and reimplanted with a silastic implant at two months, and examined at four months. Group five animals were implanted with a

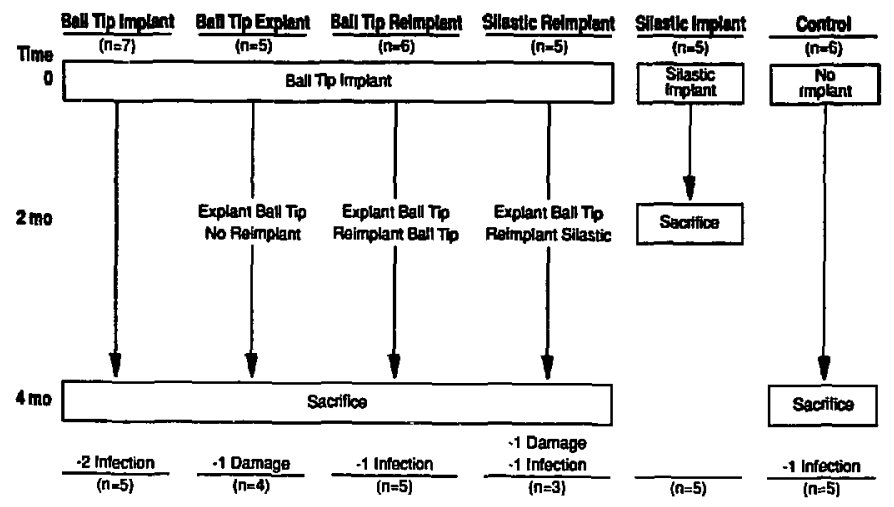

Fig. 2. Experimental design illustrating experimental groups, time course of study, number of animals used and from which groups subjects were excluded. 
silastic implant and examined at two months. Contralateral non-implanted ears served as controls.

The surgical approach was identical for both implantation and explantation of the electrodes. Animals were anesthetized with ketamine $(40 \mathrm{mg} / \mathrm{kg})$ and xylazine $(10 \mathrm{mg} / \mathrm{kg})$. Both pre- and post-operative prophylactic antibiotic (chloramphenicol) was provided. The cochlea was exposed via a post-auricular approach. The middle ear and round window membrane were free of signs of infection in all subjects used. Electrodes were placed, under direct visualization, approximately $2 \mathrm{~mm}$ through the round window membrane into the scala tympani. This resulted in placement immediately beneath the basilar membrane in the hook region of the cochlea. The electrode was then anchored to the bulla wall with Durelon, making certain that no cement entered the middle ear. During explantation a fibrous sheath surrounding the electrode was occasionally noted and care was taken to insure that it was minimally disturbed.

At the conclusion of the implanted period, each animal was deeply anesthetized with sodium pentobar- bital, systemically perfused with phosphate buffer followed by fixative containing $4 \%$ paraformaldehyde in phosphate buffer, and decapitated. Temporal bones were quickly removed and the cochleae received intrascaler perfusion with the same fixative. The cochleae were immersed in fixative overnight and then post-fixed for 1 hour in $1 \%$ osmium tetroxide. Following decalcification in $4 \%$ buffered EDTA, the cochleae were dehydrated and embedded in Embed-812 epoxy resin. Mid-modiolar sections of $6 \mu \mathrm{m}$ thickness were cut and stained for histological analysis. The average number of viable spiral ganglion cells in the seven regions of the cochlear spiral was determined in each cochlea from five representative mid-modiolar sections, as described previously (Jyung et al. 1989; Zappia et al. 1989). Cross sectional areas of each canal of Rosenthal were measured using the previously described system by Jyung et al. (1989) to calculate spiral ganglion cell densities. A value was obtained for each Rosenthal's canal from apex-to-base (A-G respectively). Area $G$ corresponds to the area immediately adjacent to the implant. Spiral ganglion densities of experimental sub-

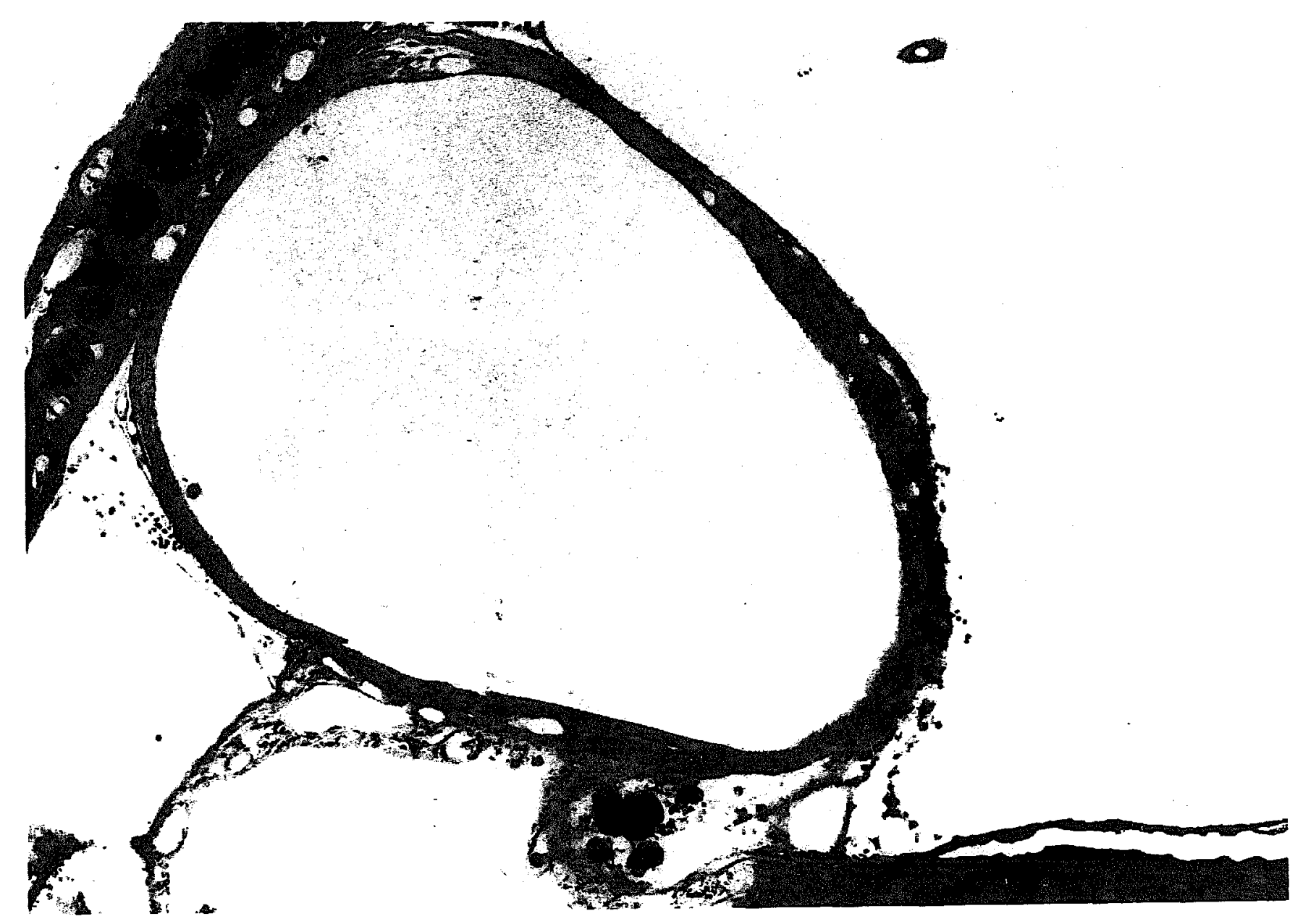

Fig. 3. Cross section of lateral scala tympani in area $G$ of an implanted and explanted cochlea. A fibrous sheath is seen with signs of new bone growth next to the lateral wall. 


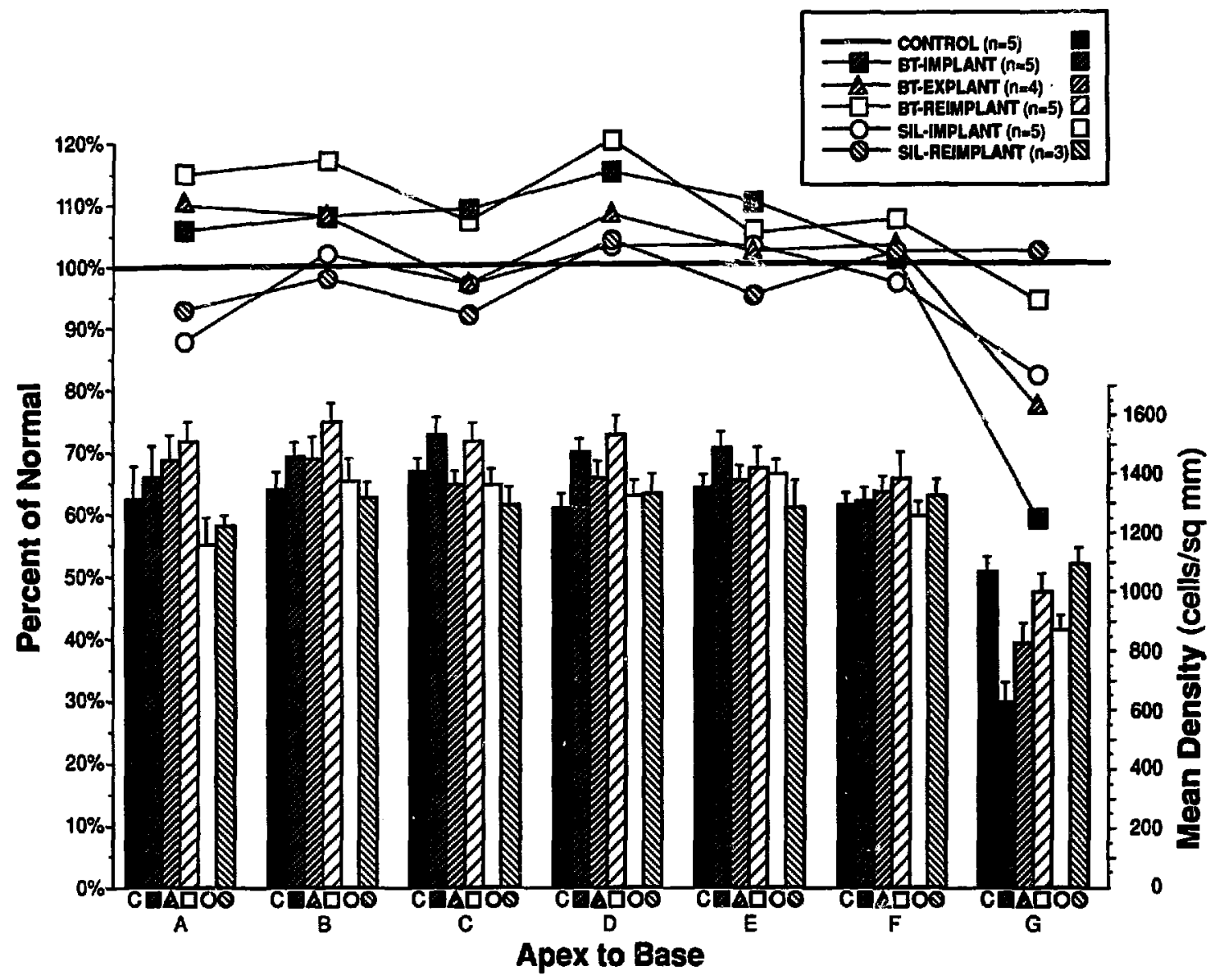

Fig. 4. Graph of mean spiral ganglion cell density. (Top): Mean spiral ganglion density shown as percent of control by experimental group in areas A-G along Rosenthal's canal. (Bottom): Mean spiral ganglion cell density values (cells/sq mm) with standard error of mean bars shown.

jects were normalized to controls and compared across experimental groups.

\section{Results}

Four of the thirty-three guinea pigs in this study were excluded due to infection. All other animals recovered fully from each operation and grew at a rate commensurate with non-implanted animals. Two additional implanted cochleae were partially damaged during histological preparation (ball-tip explant group, silastic reimplant group) and were discarded.

All cochleae were examined for organ of Corti changes at the light microscopic level and spiral ganglion cells were assessed quantitatively. Hair cell loss and organ of Corti damage was noted in 5 of 27 subjects in the basal turn (area G). These five animals were divided among four of the five groups. Of the animals excluded because of infection and in which the infection had entered into the cochlea, major hair cell loss, inflammatory reaction, and calcification was observed. A fibrous sheath was also occasionally observed (Fig. 3). When present it was always observed at the area of electrode insertion in the basal turn beneath the organ of Corti (area G) and extended beyond the round window membrane into the middle ear space. There was some irdication of osseous reaction that was restricted to the region of the lateral wall. This occurred in animals in both the ball-tip and silastic implant groups.

The mean spiral ganglion cell density values for each area (A-G) of all experimental groups and controls are shown in Fig. 4. In addition, spiral ganglion cell densities of all groups of implanted subjects were normalized to non-implanted control values according to area (A-G). A repeated measures analysis of variance (BMDP statistical package, Statistical Software Incorporated, Los Angeles, CA) was used to statistically compare mean spiral ganglion cell densities as a function of experimental implant groups. The repeated measures analysis tests for differences based on experimental implant type, as well as location within the cochlea (areas A-G). No significant differences in mean spiral ganglion densities were noted between the experimental implant groups and controls, based on implant type $(P>0.10)$.

There were decreases in spiral ganglion density in the base of some cochleae as compared to controls. This correlated with the loss of hair cells and was 
pronounced in the $\mathbf{G}$ region of three different groups: ball-tip implant ( 3 animals), ball-tip explant ( 1 animal), and the silastic implant group (1 animal). It should be noted that these three groups were subjected to less overall surgical trauma than the other two groups (the groups that had electrodes reimplanted). No significant decreases in spiral ganglion cells were noted in the apices of any of the experimental groups. One animal in the silastic implant group did show apical spiral ganglion cell loss, but it was not selective, as there were also decreased densities in the base.

\section{Conclusion}

The need for replacing cochlear prostheses will increase for a variety of reasons. As younger patients are added to the implant candidate pool, the lifespan of current devices may be exceeded by the life of the patients. With rapid advancements in cochlear prosthesis technology one can hope that replacement may be desired for purposes of system upgrading. In addition, with longer implant duration, occasional device malfunction or failure can be anticipated. When considering replacement of a cochlear prosthesis the added risk of reimplantation must be considered, especially when the existing device is functional.

Of the variety of factors that may impede reimplantation, bone formation associated with the initial implant would be quite significant. While new bone growth does not appear to be associated with most implant procedures, numerous reports of surgically induced bone formation exist. One might therefore anticipate some cases of new bone formation with cochlear implantation. Our study reflected this, with minor ossification observed in a few animals. In our relatively short term study, this did not present an impediment or increased risk to reimplantation.

Currently little is known about the risk that surgical reimplantation poses to surviving spiral ganglion cells. Obviously any surgical risk would be magnified by the number of procedures performed. On the basis of this investigation we conclude that in the guinea pig, using non-stimulated electrodes, reimplantation can be $\cdot r$ formed with little additional risk to spiral ganglion cells. However, it remains unclear as to whether this is a species specific finding, and warrants further investigations involving the primate. In addition, longer follow up may be necessary to detect specific pathological changes associated with reimplantation.

\section{Acknowledgements}

This work was supported by NIH grant NS 21440 and FDA Contract 223-87-6028. The authors gratefully acknowledge J. Horn, D. Moscow and C. Sheridan for technical assistance, J. Warner for statistical assistance and Denise D. Dyson for assistance in manuscript preparation.

\section{References}

Hartshorn, D.O., Miller, J.M. and Altschuler, R.A. (1991) Protective effect of electrical stimulation in the deafened guinea pig cochlea. Otolaryngol. Head Neck Surg. 104(3), 311-319.

Jyung, R.W., Miller, J.M. and Cannon, S.C. (1989) Evaluation of eighth nerve integrity using the electrically evoked middle latency response. Otolaryngol. Head Neck Surg. 101(6), 670-682.

Leake, P.A., Snyder, R.L., Chambers, P.L., Hradek, G.T., Rebscher, S.J., Bates, G.J. and Marks, D.R. (1989) Consequences of chronic electrical stimulation in an animal model of congenital profound hearing loss. Abst. Assoc. Res. Otol. 12, 268.

Leake P.A., Rebscher, S.J. and Aird, D.W. (1985) Histopathology of cochlear implants: safety considerations. In: R.A. Schindler and M.M. Merzenich (Eds.), Cochlear Implants, Raven Press, New York, pp. 55-64.

Leake-Jones, P.A. and Rebscher, S.J. (1983) Cochlear pathology with chronically implanted scala tympani electrodes. In: C.W. Parkins and S.W. Anderson (Eds.), Cochlear Prosthesis: An International Symposium, Ann. N.Y. Acad. Sci. 405, pp. 203-205.

Lousteau, R.J. (1987) Increased spiral ganglion cell survival in electrically stimulated, deafened guinea pig cochleae. Laryngoscope 97, 837-842.

Miller, J.M., Altschuler, R.A., Carlisle, L., Dengerink, H.A., Kemink, J. and Sutton, D. (1987) Cochlear prosthesis: histological observation on reimplaniations in the monkey. Abst. Assoc. Res. Otol. $10,54$.

Miller, J.M., Altschuler, R.A., Niparko, J.K. Hartshorn, D.O., Helfert, R.H. and Moore, J.K. (1991) Deafness induced changes in the central nervous system and their reversibility and prevention. In: IV International Conference on the Effects of Noise on the Auditory System (in press).

Otte, J., Schuchnecht, H.F. and Kerr, A.G. (1978) Ganglion cell populations in normal and pathological human cochlea: Implications for cochlear implantation. Laryngoscope 88, 1231-1246.

Pfingst, B.E., Glass, I., Spelman, F.A. and Sutton, D. (1985) Psychophysical studies of cochlear implants in monkeys: Clinical implications. In: R.A. Schindler and M.M. Merzenich (Eds.), Cochlear Implants, Raven Press, New York, pp. 305-322.

Schindler, R.A. and Bjorkroth, B. (1979) Traumatic electrode implantation. Laryngoscope 89, 752-758.

Schindler, R.A. (1976) The cochlear histopathology of chronic intracochlear implantations. J. Laryngol. Otol. 90, 445-457.

Schindler, R.A. and Merzenich, M.M. (1974) Chronic intracochlear electrode implantation: cochlear pathology and acoustic nerve survival. Ann. Otol. Rhinol. Laryngol. 83, 202-215.

Webster, M. and Webster, D.B. (1981) Spiral ganglion neuron loss following organ of Corti loss: A quantitative study. Brain Res. 212, 17-30.

Zappia, J.J. and Altschuler, R.A. (1989) Evaluation of the effect of ototopical neomycin on spiral ganglion cell density in the guinea pig. Hear. Res. 40, 29-38. 